\title{
Automatic identification of familiar faces
}

\author{
Kyunghun Jung • Eric Ruthruff • Nicholas Gaspelin
}

Published online: 8 June 2013

(C) Psychonomic Society, Inc. 2013

\begin{abstract}
Much research has suggested that facial identification has some characteristics of automaticity, in that it is rapid, nonconscious, and mandatory. However, little research has tested whether it can occur even if attention is already devoted to the demanding central processes of another task. In the present study, we addressed this type of automaticity using the psychological refractory period paradigm. In Experiments 1 and 2, participants successfully identified familiar faces, even while they were engaged with another task, indicating the automaticity of familiar-face identification. In Experiments 3 and 4, however, participants could not identify unfamiliar faces as automatically as they could identify the familiar faces. We concluded that automatic face identification is possible, but dependent on prior familiarity.
\end{abstract}

Keywords Face identification - Automaticity .

Psychological refractory period paradigm

Human faces provide information critical to our survival and reproductive fitness, such as identity, emotion, eye gaze, and attractiveness. Facial identity allows us to differentiate between friends and foes, facial emotion provides a cue to predict potential threat from others, eye gaze tells us where the person is attending (Langton, Watt, \& Bruce, 2000), and facial attractiveness signals genetic quality and phenotypic condition (Grammer, Fink, Møller, \& Thornhill, 2003). Given the high importance of facial information processing, it has been considered a strong candidate for automaticity, despite a high degree of computational complexity (for a review, see Palermo \& Rhodes, 2007). Furthermore, facial information processing is extensively practiced (e.g., we identify faces of friends and colleagues many times per

K. Jung $(\bowtie) \cdot$ E. Ruthruff $\cdot$ N. Gaspelin

Department of Psychology, University of New Mexico,

MSC03 2220, Albuquerque, NM 87131-1161, USA

e-mail: khjung@unm.edu day), which might also promote automatic processing (Maquestiaux, Laguë-Beauvais, Ruthruff, \& Bherer, 2008).

In their seminal work, Schneider and Shiffrin (1977) proposed that an automatic process has the following properties: It (1) occurs relatively rapidly, (2) proceeds without conscious awareness, and (3) is mandatorily initiated whenever a triggering stimulus is present (see also Shiffrin \& Schneider, 1977). More directly related to the present study, they also proposed that an automatic process should require few or no attentional resources. In other words, an automatic mental process is not impeded by other demanding mental processes occurring simultaneously (see also Norman \& Bobrow, 1975; Schneider \& Fisk, 1982). The present research focuses on whether facial identification meets the latter criterion for automaticity.

Previous studies have shown that facial identification has some of the characteristics of automaticity. For example, rapid facial identification is supported by the findings that facespecific magnetoencephalography responses occur only $170 \mathrm{~ms}$ after face onset, and that this early activity is correlated with successful facial identification (Liu, Harris, \& Kanwisher, 2002). Studies examining the skin conductance response of prosopagnosic patients have revealed that such patients can successfully identify familiar faces, despite the absence of conscious awareness (Bauer, 1984; see also Tranel \& Damasio, 1985). Similarly, normal participants are able to preconsciously identify familiar faces that are presented briefly (17 ms) and followed by a backward mask (Stone \& Valentine, 2004). In addition, facial stimuli have been shown to produce negative priming effects - that is, impaired recognition of a target that was recently ignored as a distractor- even when there was no incentive to process them, suggesting the mandatoriness of facial identification (Khurana, Smith, \& Baker, 2000; see also Boutet, Gentes-Hawn, \& Chaudhuri, 2002; Lavie, Ro, \& Russell, 2003).

Despite this evidence that facial identification shows various signs of automaticity, it has remained unclear from previous studies whether facial identification can occur even 
if attention is already devoted to the demanding central processes of another task. One line of studies has indicated that facial identification is nonautomatic, requiring access to attentional resources. For example, Palermo and Rhodes (2002) found that holistic ${ }^{1}$ facial processing of a target face was impaired when participants were instructed to identify two other, peripheral faces presented simultaneously. This result suggests that, with divided attention, facial identification is incomplete. Similarly, Jackson and Raymond (2006) used the attentional blink ( $\mathrm{AB}$ ) paradigm ${ }^{2}$ to investigate the interaction between attention and facial identification. They found an $\mathrm{AB}$ effect when the faces (T2) were unfamiliar to participants, but not when the faces were familiar. This finding suggests that the identification of unfamiliar faces requires attentional resources.

Another line of studies has suggested that facial identification can occur without certain attentional resources. In their influential work, Bruce and Young (1986) argued that facial identification can operate in parallel with other types of facial analyses, such as expression recognition or facial speech understanding. This conclusion has been supported by neurophysiological evidence that different neurologic systems are responsible for different facial information processes (Allison, Puce, Spencer, \& McCarthy, 1999). Their hypothesis has also been supported by behavioral experiments. For example, Bruce (1979) showed that when participants were instructed to find famous politicians (targets) within a sequence of familiar and unfamiliar distractor faces, the effects of distractor-target semantic category similarity (i.e., whether the distractor faces were also politicians) was independent of the effect of distractor-target visual similarity (physical resemblance). Bruce concluded that facial identification and other perceptual assessments of faces can occur in parallel. Finally, using the AB paradigm, Awh et al. (2004) observed a result opposite to that of Jackson and Raymond (2006) — no AB effect from unfamiliar T2 faceseven when the same $\mathrm{T} 1$ task (digit discrimination) produced an $\mathrm{AB}$ effect on a letter discrimination $\mathrm{T} 2$ task. The absence of an $\mathrm{AB}$ effect for unfamiliar $\mathrm{T} 2$ faces suggests that facial

\footnotetext{
${ }^{1}$ Previous studies have shown that facial processing is holistic rather than featural, especially when compared to other types of object processing (e.g., Farah, Wilson, Drain, \& Tanaka, 1998). That is, facial features are represented in an integrated form, rather than being processed independently from one another. This view has been supported by studies showing that the recognition of facial features is dependent on the spatial relationship between the target feature (e.g., nose) and other features (e.g., eyes). This holistic facial processing is considered to be the basis of successful facial identification.

${ }^{2}$ When subjects are instructed to identify two targets in a rapid serial visual presentation, they typically show impaired detection/identification of the second target (T2) when it is presented within $200-400 \mathrm{~ms}$ of the first target (T1). The reduced $\mathrm{T} 2$ performance (an attentional blink, or $\mathrm{AB}$, effect) is often attributed to a temporary depletion of attentional resources, caused by $\mathrm{T} 1$ processing.
}

identification does not require the specific attentional resources required to perceive and encode $\mathrm{T} 1$.

The preceding studies mainly focused on whether facial processing can operate in parallel with other perceptual processes. However, one question thus far neglected is whether facial identification requires central attentional resources. This is a critical issue, because central attentional resources represent a major bottleneck in information processing in multitasking situations (Pashler, 1984).

\section{The PRP paradigm: A tool for assessing whether a mental process requires central attentional resources}

To study whether a certain mental process requires central attentional resources, researchers often utilize the psychological refractory period (PRP) paradigm. In this paradigm, participants make speeded responses to two tasks (Task 1 and Task 2). The critical independent variable is the interval between the onsets of the Task-1 and Task- 2 stimuli, called the stimulus onset asynchrony (SOA). The dependent variables are the response time to Task 1 (RT1) and Task 2 (RT2), although RT2 is the main subject of analysis. At long SOAs, such as $900 \mathrm{~ms}$, participants typically can complete Task 1 even before the Task-2 stimulus appears. Consequently, the processing of the two tasks does not overlap and can be completed largely independently. This long-SOA condition provides a performance baseline for the two tasks. The main question in the PRP paradigm is how much interference will occur at short SOAs (e.g., $100 \mathrm{~ms}$ ), at which task processing does overlap. The ubiquitous pattern observed in previous PRP experiments is a considerable slowing of RT2 at short SOAs, known as the PRP effect.

The PRP effect is typically explained by the central bottleneck model (e.g., Pashler, 1984; Ruthruff, Johnston, \& Remington, 2009; for a review, see Lien, Ruthruff, \& Johnston, 2006). The central bottleneck model decomposes the tasks into three discrete processing phases: perceptual processes, central processes, and response execution. It postulates that central processes (such as decision-making or response selection) cannot occur simultaneously for different tasks. At short SOAs, a consequence of this central processing bottleneck is a delay, whereby Task- 2 central processes must wait for Task-1 central processes to finish. The waiting period is often called cognitive slack (see the dotted line in Fig. 1).

The central bottleneck model is supported by many studies (e.g., Lien, Ruthruff, \& Johnston, 2006; Pashler, 1984; Ruthruff et al., 2009), suggesting that there is some attentional resource that cannot be simultaneously shared among central processes. This resource is referred to as "central attention" because early perceptual processes and later response processes generally do not require it. Given this model, "locus-of- 


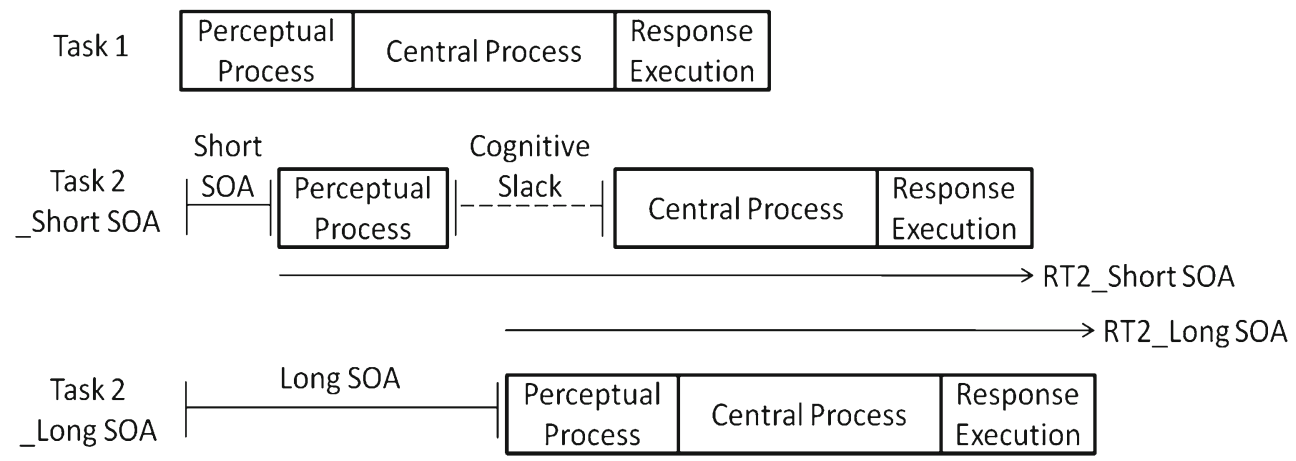

Fig. 1 Illustration of the central-bottleneck model and how it produces dual-task interference (substantial slowing of Task-2 responses at short stimulus onset asynchronies [SOAs]). The key assumption of this model is that central processes occur for only one task at a time; that is, the Task- 2 central process waits until the Task-1 central process is finished. At short SOAs (middle panel), this central-bottleneck limitation produces a period of cognitive

slack" logic can be used to determine whether a target process (e.g., face identification) in Task 2 is automatic; that is, whether it can occur simultaneously with Task-1 central process (see Pashler, 1984). To use locus-of-slack logic, one first needs to manipulate the difficulty of the target process, increasing its duration. For example, in the present study, we manipulated the difficulty of facial identification by making the target faces look more or less similar to each other. Difficulty effects on RT2 depend critically on whether the target process is automatic. If the target process is automatic, then it can co-occur with the Task-1 central process. In this case, any lengthening of the target process can be absorbed into the period of cognitive slack at short SOAs, as is shown in panel A of Fig. 2. Thus, the difficulty effect on RT2 should decline or even disappear at short SOAs, while remaining substantial at long SOAs - an underadditive interaction of difficulty and SOA. ${ }^{3}$ On the other hand, if the target process is not automatic (i.e., needs central attentional resources), then it can take place only after completion of the Task-1 central process (see panel B of Fig. 2). Therefore, the difficulty effect will be full-blown at short SOAs, just as it is at long SOAs (a data pattern known as additivity). Although it is simplest to imagine the two extreme outcomes described above (complete automaticity or complete nonautomaticity), of course intermediate outcomes are also possible, resulting in partial underadditivity implying partial automaticity (see the present Exps. 3 and 4).

Studies employing this locus-of-slack logic have sometimes produced strong reductions in difficulty effects at short SOAs (i.e., underadditivity)—indicating automaticity—and

\footnotetext{
${ }^{3}$ To eliminate the difficulty effect at short SOAs, the cognitive slack must be relatively long, compared to the duration of the target process in the difficult condition; that is, the target process must always finish before the period of cognitive slack is over.
}

slack in Task 2, indicated by the dotted line. This central bottleneck is assumed to be the main source of dual-task interference. On the other hand, at long SOAs (bottom panel), Task- 2 central process can start immediately after the perceptual process, without any waiting, and therefore without any slowing of Task-2 responses. RT1, Task-1 RT; RT2, Task-2 RT; SOA, stimulus onset asynchrony

have sometimes produced equal difficulty effects across SOAs (i.e., additivity) - indicating nonautomaticity. For example, automaticity has been reported with relatively simple tasks such as letter identification (Pashler \& Johnston, 1989), and word reading (at least, in older adults and better younger readers; Ruthruff, Allen, Lien, \& Grabbe, 2008). Meanwhile, nonautomaticity has been reported for many other mental processes, such as mental rotation (Ruthruff, Miller, \& Lachmann, 1995), box-width judgments (Johnston \& McCann, 2006), reading words aloud (O’Malley, Reynolds, Stolz, \& Besner, 2008), depressing a vehicle's brake pedal (Levy, Pashler, \& Boer, 2006), facial emotion perception (Tomasik, Ruthruff, Allen, \& Lien, 2009), and facial attractiveness judgments (Jung, Ruthruff, Tybur, Gaspelin, \& Miller, 2012).

\section{The present study}

To briefly summarize research on the automaticity of facial identification, whereas there is a general consensus that facial identification (1)occurs relatively rapidly, (2)proceeds without conscious awareness, and (3)is mandatory, it remains unclear whether facial identification can bypass the central attentional bottleneck. In the present study, we addressed this latter issue, which we refer to simply as "automaticity" for ease of discussion, using the PRP paradigm with locus-of-slack logic.

This study also has important implications for automaticity research more generally. Previous studies suggest that this type of automaticity - bypassing the central bottleneck -is the exception rather than the rule. That is, only a few simple and highly practiced processes such as letter identification have thus far been shown to be automatic (Pashler 


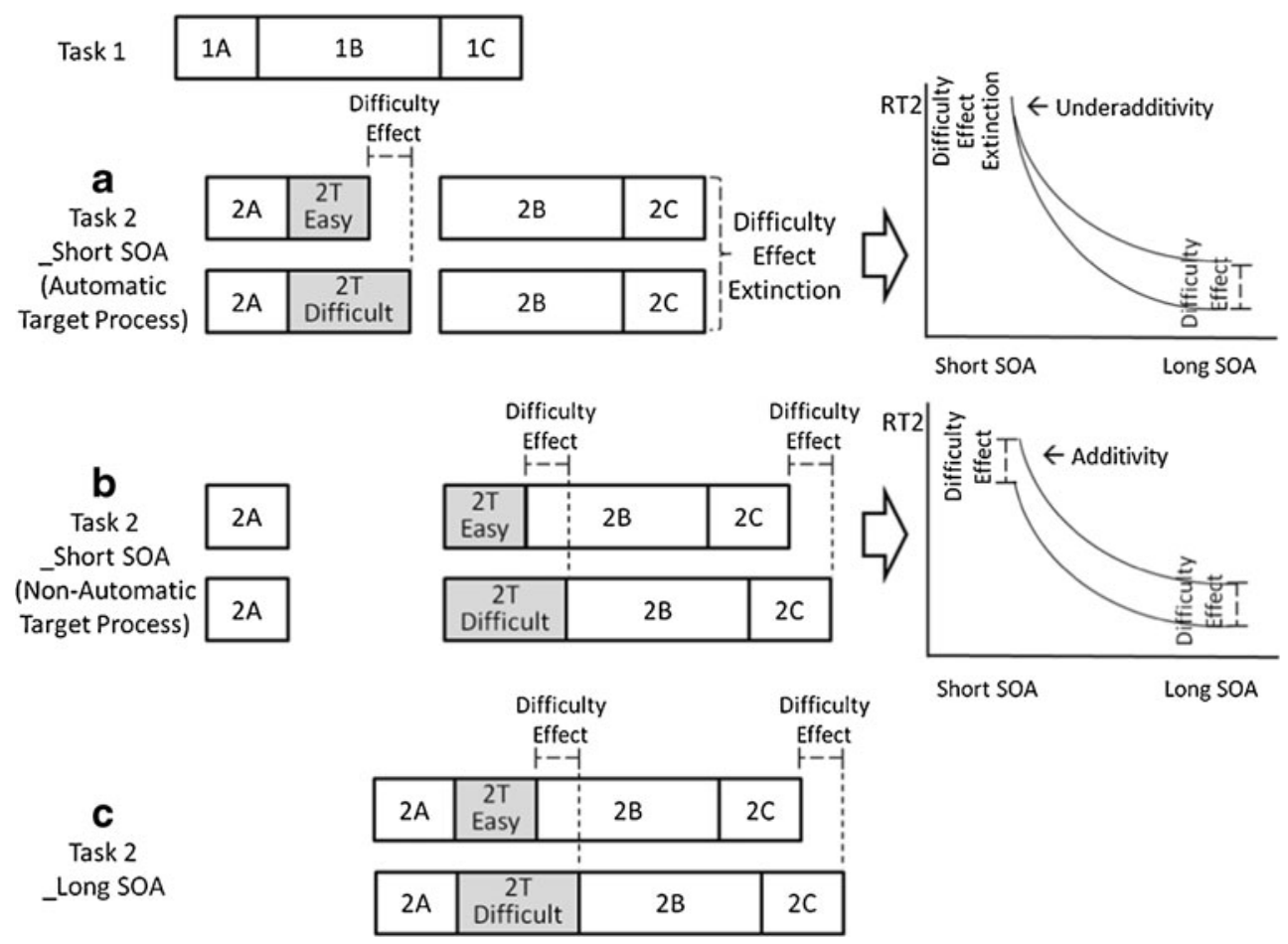

Fig. 2 (A) Predictions regarding the Task-2 difficulty effect at short SOAs if the target process in Task $2(2 \mathrm{~T})$ does not require central attentional resources (i.e., operates automatically). In this case, the difficulty effect is absorbed into cognitive slack. As a result, the difficulty effect on the Task-2 response time (RT2) would markedly decrease at short SOAs (producing underadditivity between SOA and difficulty). (B) Predictions regarding the Task-2 difficulty effect at short SOAs if $2 \mathrm{~T}$ does require central attentional resources (i.e., does not operate automatically). In this case, $2 \mathrm{~T}$ can occur only after $1 \mathrm{~B}$ is finished. As a result, any lengthening of the $2 \mathrm{~T}$ will increase the

\& Johnston, 1989). Facial identification is far from a simple process because faces are very complex, involving many different features that can change over time. Therefore, there is reason to be skeptical that facial identification is indeed automatic. However, as noted above, facial identification has high evolutionary importance and is also highly practiced (indeed, we start to learn faces even before we learn letters) making it a plausible candidate for automaticity.

In Experiments 1 and 2, we assessed whether identification of familiar celebrity faces is automatic. In Experiments 3 and 4, we assessed whether facial identification of much less familiar faces is automatic.

\section{Experiment 1}

To test whether facial identification of familiar faces is automatic, we used face pictures from three famous actors. Facial identification, by its very nature, requires a priori knowledge of the faces. Therefore, celebrities' faces, which are known to most participants, have been widely used in overall RT2 at short SOAs (producing additivity between SOA and difficulty). (C)Predictions regarding the Task-2 difficulty effect at long SOAs. The difficulty effect will be fully reflected in RT2 at long SOAs, regardless of the automaticity of $2 \mathrm{~T}$, because there is no cognitive slack into which the difficulty effect can be absorbed. 1A, 1B, and 1C are the perceptual, central, and response execution processes of Task 1 , respectively; $2 \mathrm{~A}, 2 \mathrm{~B}$, and $2 \mathrm{C}$ are the corresponding processes of Task 2 . $2 \mathrm{~T}$ is the target process in Task 2. RT2 is the response time of Task 2, and SOA is the stimulus onset asynchrony

facial identification studies (e.g., Jackson \& Raymond, 2006; Lavie et al., 2003).

In the present PRP paradigm, we employed two tasks: a tone discrimination task (Task 1) and a facial identification task (Task 2). On each trial, their stimulus onsets were separated by a variable SOA $(100,300,500$, or $900 \mathrm{~ms})$. The purpose of Task 1 is simply to engage central attentional resources, so that they are unavailable to Task 2 for some extended period of time. We manipulated Task-2 difficulty by modifying each original celebrity face to look more similar to one of the other celebrities, using morphing software (see the Method section). Automatic face identification predicts that the Task-2 difficulty effect should be smaller at short SOAs than long SOAs, or even disappear entirely.

Method

Participants A group of 48 undergraduate students from the University of New Mexico participated in exchange for course credit. All self-reported normal or corrected-to-normal visual acuity. 
Apparatus and stimuli For the tone-discrimination task (Task 1), one of two tones (1.0 or $1.5 \mathrm{kHz})$, was presented through headphones. For the facial identification (Task 2), a picture of one of three celebrities (Tom Cruise, Matt Damon, and Brad Pitt) was presented on a 19-in. high-resolution color monitor. We collected 4 pictures for each of the celebrities from the internet, for a total of 12 . The facial pictures were cropped so that they just barely included the hair, ears, and the top of the shoulders. At a typical viewing distance of $55 \mathrm{~cm}$, the faces subtended a visual angle of about $9^{\circ} \times 18^{\circ}$ (measured from cheek to cheek and from the middle of the forehead to the bottom of the chin).

The stimuli for the easy condition consisted of the 12 original facial pictures, whereas the stimuli for the difficult condition consisted of 12 morphed images. Morphed images were created by morphing one of the original pictures of a celebrity with a picture of one of the other celebrities in a 70 versus 30 mixture, using Magic Morph software (www.effectmatrix.com/morphing/). For each actor, 32 possible morphed images were created (4 original pictures $\times 8$ pictures of the other actors); from these 32 pairs, we chose the four that had the most similar facial dimensions. Examples of the stimuli are shown in Fig. 3. The morphed images could still be reliably identified as the intended celebrity (i.e., accuracy was high, as is shown in Table 1), but they required more time to identify, as is shown in our experimental data.

Procedure At the beginning of each trial, a black fixation cross was presented on a white background for 1,000 ms. After the fixation disappeared, the Task-1 stimulus, either a low-pitched or high-pitched tone, was presented for $200 \mathrm{~ms}$. Participants were asked to indicate quickly whether the tone was low or high in pitch by pressing either the horizontally adjacent " $X$ " or " $Z$ " key, respectively, using their left middle or index finger. After one of four different SOAs (100, 300, 500 , or $900 \mathrm{~ms}$ ), the Task-2 stimulus, either an original (easy condition) or morphed face (difficult condition), was presented on the screen. It remained until a response was registered or until the trial timed out (after $3 \mathrm{~s}$ ). Participants were asked to identify whether the face was Tom Cruise, Matt Damon, or Brad Pitt by pressing a corresponding key as quickly as possible. These three celebrities were assigned to the horizontally adjacent "V," "B," and "N" keys, respectively. Participants used their right index, middle, and ring fingers, respectively, for those three keys.

After each trial, participants received feedback regarding both tasks. If a response was correct, RT1 and RT2 in milliseconds were presented in blue letters. For incorrect or slow responses (longer than 1,500 ms for RT1 or longer than 3,000 ms for RT2), a "Wrong" or "Too slow" error message was presented in red. Task-1 feedback was presented on the left side of the screen and Task-2 feedback on the right side, simultaneously for 1,000 ms.
Before the main experimental blocks, all participants completed two practice blocks to learn the two component tasks. In the first practice block (24 randomly selected trials from the main experimental trials of the easy condition), participants performed Task 1 only, ignoring the Task-2 stimulus. In the second practice block (another 24 randomly selected trials), participants performed Task 2 only, ignoring the Task-1 stimulus. Each participant then performed 12 main experimental blocks of 32 trials each.

Design During the experimental blocks, all combinations of the four different SOAs and the two Task-1 stimuli (high or low-pitched tone) were selected twice for each of the 24 face pictures (12 original and 12 morphed), yielding 384 trials in total for each participant $(4 \times 2 \times 2 \times 24=384)$. These trials were randomly distributed in the 12 main experimental blocks.

Results and discussion

The first experimental block and the first two trials of each subsequent block were regarded as warm-up trials and not included in the data analysis. Trials were excluded if either RT was below $200 \mathrm{~ms}$ or above $1,500 \mathrm{~ms}$ ( $3 \%$ of RT $1 \mathrm{~s}$ and $2 \%$ of RT2s). Trials were also excluded from the RT data analysis if they contained an incorrect response to Task 1 (3\% of the trials) or Task 2 ( $7 \%$ of the trials). We removed the data from one participant with low Task-1 accuracy (below our criterion of $85 \%$ ). We also discarded data from three additional participants with low Task-2 accuracy in the easy condition (below our criterion of $80 \%$ ). After this data selection process, 44 out of 48 participants remained for further data analysis. The resulting mean RTs and proportions of errors (PEs) for Task 1 and Task 2 are shown in Table 1.

$R T$ analysis $\mathrm{A}$ repeated measures analysis of variance (ANOVA) was conducted with the factors of SOA and Task2 Difficulty on RT1 and RT2. When assumptions of sphericity were violated, $p$ values were adjusted using the GreenhouseGeisser or Huynh-Feldt correction according to their epsilon values. RT1s were consistent across $\operatorname{SOAs}[F(3,129)=1.83$, $\left.p<.17, \eta_{\mathrm{p}}{ }^{2}=.41\right]$. As is shown in Fig. 4 (upper panel), the mean RT2 increased by $200 \mathrm{~ms}$ as SOA decreased, reflecting dual-task interference at short SOAs $[F(3,129)=274.61$, $\left.p<.01, \eta_{\mathrm{p}}{ }^{2}=.87\right]$. According to the central bottleneck model, the RT2 increase at the shortest SOA by $200 \mathrm{~ms}$ - the PRP effect - occurred because Task- 2 central process waits for central attentional resources to become available following the completion of Task- 1 central processes.

Most importantly, the automatic facial-identification hypothesis predicts that facial identification on Task 2 can cooccur with Task-1 central processes (i.e., proceeds during the period of cognitive slack). If so, there should be sufficient time to identify both the easy and difficult faces during 

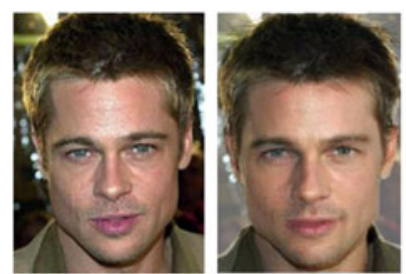

Fig. 3 Examples of stimuli used in Experiments 1, 2, and 4. The left images of each pair are the original pictures (easy condition), and the right images are morphed images (difficult condition). The three image pairs shown are facial images of, from the left, Brad Pitt from

the relatively long cognitive slack (i.e., $200 \mathrm{~ms}$ ). As a result, we should see almost no Task-2 difficulty effect at the shortest SOA (see panel A of Fig. 2), which is often called "absorption into cognitive slack." However, we would always see the full difficulty effect at longer SOAs, where there is almost no cognitive slack. Consistent with this automatic facial-identification hypothesis, Fig. 4 (upper panel) shows a remarkable decrease in the Task-2 difficulty effect at the shortest relative to the longest SOA. This pattern was confirmed by the significant interaction between Task-2 difficulty (RT2 in the difficult condition-RT2 in the easy condition) and SOA $\left[F(3,129)=6.49, p<.01, \eta_{\mathrm{p}}{ }^{2}=.13\right]$; the difficulty effects were $2,30,31$, and $37 \mathrm{~ms}$ at the 100-, 300-, $500-$, and 900-ms SOAs, respectively. The difficulty effect of $2 \mathrm{~ms}$ at the shortest SOA $(95 \% \mathrm{CI}:[-12,17])$ was significantly smaller than the 37 -ms difficulty effect at the longest SOA $[F(1$,

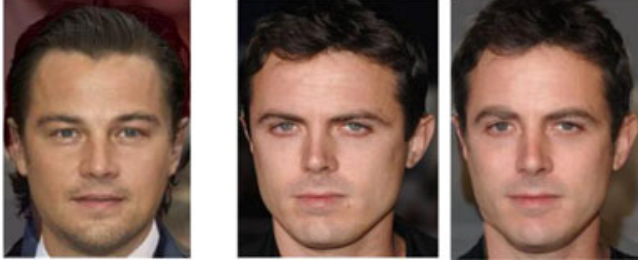

Experiment 1 (morphed with Matt Damon), Leonard DiCaprio from Experiment 2 (morphed with Keanu Reeves), and Casey Affleck from Experiment 4 (morphed with Jeremy Northam). The morphing ratio was 70 (original) versus 30 (mixture)

$\left.43)=17.09, p<.01, \eta_{\mathrm{p}}{ }^{2}=.28\right]$ and was not significantly greater than $0 \mathrm{~ms}\left[F(1,43)=0.11, p=.74, \eta_{\mathrm{p}}^{2}=.01\right]$. On the basis of locus-of-slack logic, the above findings suggest that facial identification can co-occur with Task-1 central processes, indicating automatic identification of familiar faces.

$P E$ analysis The mean Task-1 PE (PE1) was affected by SOA $\left[F(3,129)=6.19, p<.01, \eta_{\mathrm{p}}{ }^{2}=.13\right]$; PE1 was .01 greater at the shortest SOA than the longest SOA. No other main effects or interactions were statistically significant for PE1. The mean Task-2 PE (PE2) was not affected by SOA $\left[F(3,129)=2.46, p=.07, \eta_{\mathrm{p}}{ }^{2}=.05\right]$. However, it was affected by the Task-2 difficulty manipulation $[F(1,43)=$ $\left.32.22, p<.01, \eta_{\mathrm{p}}{ }^{2}=.45\right]$; PE2 was .05 in the easy condition, and it increased to .08 in the difficult condition. The difficult effects on PE2 were $.01, .03, .03$, and .03 at the four SOAs,

Table 1 Mean response times (RTs, in milliseconds) and proportions of errors (PEs) for Task 1 and Task 2 as a function of Task-2 difficulty and stimulus onset asynchrony (SOA, in milliseconds) in all experiments

\begin{tabular}{|c|c|c|c|c|c|c|c|c|c|c|}
\hline \multirow{3}{*}{ EX } & \multirow{3}{*}{ Task } & \multirow{3}{*}{ Task 2 Difficulty } & \multicolumn{8}{|c|}{ SOA } \\
\hline & & & \multicolumn{2}{|l|}{100} & \multicolumn{2}{|l|}{300} & \multicolumn{2}{|l|}{500} & \multicolumn{2}{|l|}{900} \\
\hline & & & RT & PE & $\mathrm{RT}$ & $\mathrm{PE}$ & RT & $\mathrm{PE}$ & $\mathrm{RT}$ & $\mathrm{PE}$ \\
\hline \multirow[t]{4}{*}{ EX 1} & \multirow[t]{2}{*}{ Task 1} & Difficult & 626 & .03 & 610 & .02 & 619 & .02 & 615 & .03 \\
\hline & & Easy & 635 & .04 & 613 & .02 & 611 & .02 & 623 & .02 \\
\hline & \multirow[t]{2}{*}{ Task 2} & Difficult & 819 & .06 & 715 & .09 & 657 & .08 & 637 & .08 \\
\hline & & Easy & 817 & .06 & 685 & .06 & 626 & .05 & 600 & .05 \\
\hline \multirow[t]{4}{*}{ EX 2} & \multirow[t]{2}{*}{ Task 1} & Difficult & 626 & .04 & 610 & .02 & 619 & .02 & 615 & .02 \\
\hline & & Easy & 635 & .03 & 613 & .03 & 611 & .03 & 623 & .02 \\
\hline & \multirow[t]{2}{*}{ Task 2} & Difficult & 822 & .09 & 708 & .10 & 670 & .10 & 624 & .08 \\
\hline & & Easy & 816 & .06 & 679 & .06 & 624 & .05 & 584 & .06 \\
\hline \multirow[t]{4}{*}{ EX 3} & \multirow[t]{2}{*}{ Task 1} & Difficult & 649 & .05 & 637 & .02 & 649 & .02 & 656 & .02 \\
\hline & & Easy & 645 & .04 & 630 & .02 & 640 & .02 & 655 & .02 \\
\hline & \multirow[t]{2}{*}{ Task 2} & Difficult & 855 & .09 & 709 & .09 & 640 & .09 & 606 & .09 \\
\hline & & Easy & 831 & .06 & 682 & .06 & 606 & .06 & 569 & .05 \\
\hline \multirow[t]{4}{*}{ EX 4} & \multirow[t]{2}{*}{ Task 1} & Difficult & 629 & .04 & 604 & .02 & 619 & .02 & 612 & .02 \\
\hline & & Easy & 617 & .03 & 605 & .02 & 608 & .02 & 615 & .02 \\
\hline & \multirow[t]{2}{*}{ Task 2} & Difficult & 813 & .07 & 672 & .07 & 618 & .07 & 583 & .08 \\
\hline & & Easy & 791 & .06 & 646 & .04 & 584 & .05 & 551 & .05 \\
\hline
\end{tabular}

EX: experiment 

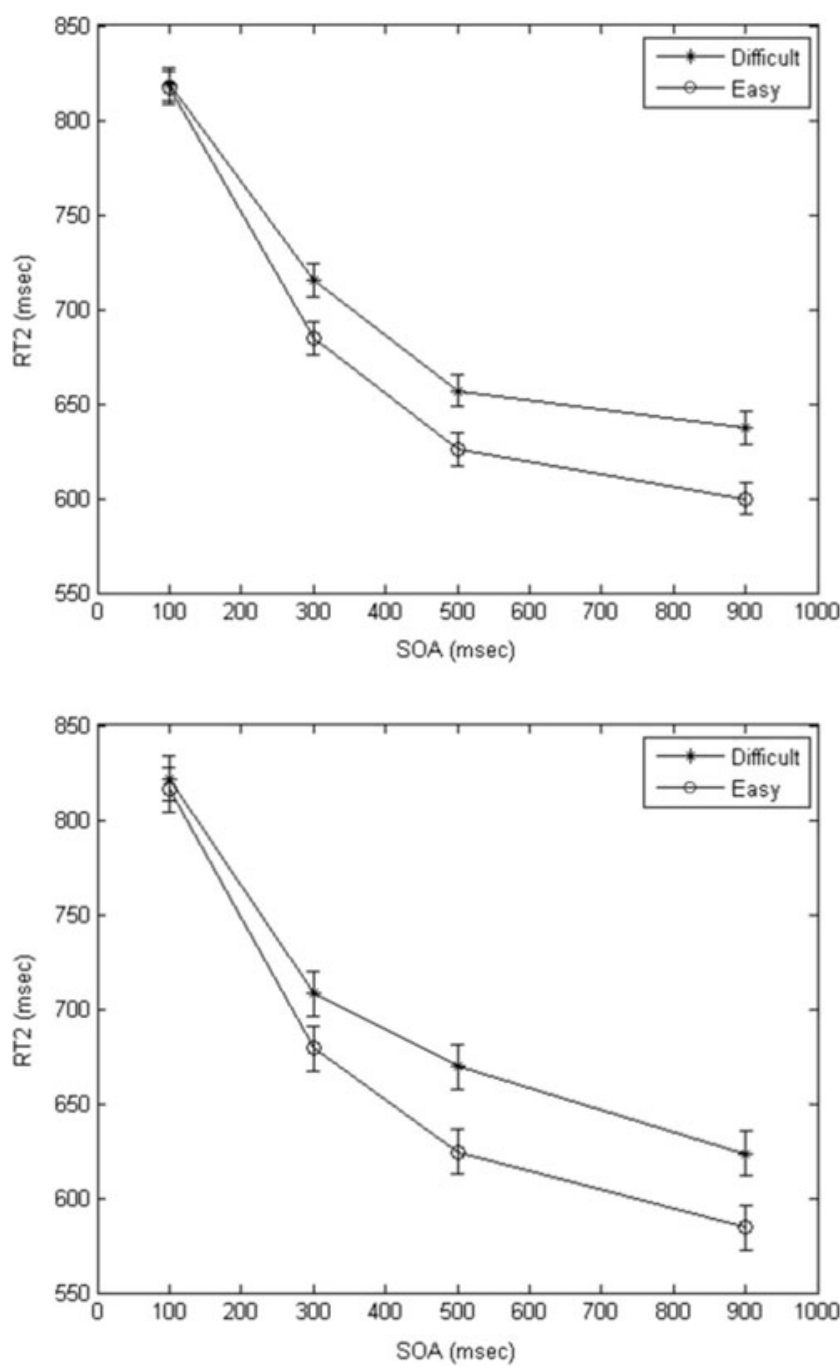

Fig. 4 Mean response times (RTs, in milliseconds) for Task 2 in Experiment 1 (upper panel) and Experiment 2 (lower panel) as a function of stimulus onset asynchrony (SOA, in milliseconds) and Task-2 difficulty

respectively. The interaction between SOA and difficulty on PE2 was not significant $\left[F(3,129)=2.43, p=.07, \eta_{\mathrm{p}}{ }^{2}=.05\right]$.

To summarize the findings of this experiment, participants identified celebrity faces while performing a concurrent tone task. According to locus-of-slack logic, if a manipulated target process is automatic, then difficulty effects should be much smaller at short SOAs than long SOAs. We observed exactly this trend, with a 2-ms difficulty effect at the shortest SOA and a 37 -ms difficulty effect at the longest SOA.

\section{Experiment 2}

It was surprising to observe underadditivity from a process as complex as facial identification. Previous PRP studies have thus far mainly shown automaticity only for relatively simple stimuli, such as letter identification (Pashler \& Johnston, 1989). Word reading is only partially automatic, except perhaps for older readers (Lien et al. 2006) and especially skilled younger readers (Ruthruff et al., 2008). Recent PRP studies using facial stimuli also suggested that facial emotion perception (Tomasik et al., 2009; but see also Shaw, Lien, Ruthruff, \& Allen, 2011) and facial attractiveness judgments (Jung et al., 2012) are not automatic. The goal of Experiment 2, therefore, was to replicate Experiment 1 using facial pictures of a different set of celebrities (Keanu Reeves, Leonard DiCaprio, and Matthew McConaughey) and to see whether the findings would generalize.

\section{Method}

Participants A group of 27 undergraduate students from the University of New Mexico participated in exchange for course credit. All self-reported normal or corrected-to-normal visual acuity.

Apparatus, stimuli, procedure, and design Experiment 2 was identical to Experiment 1, except that the pictures were of three different celebrities (Keanu Reeves, Leonard DiCaprio, and Matthew McConaughey).

\section{Results and discussion}

Data quality control was similar to that for Experiment 1 . Trials with RT1 or RT2 below $200 \mathrm{~ms}$ or above $1,500 \mathrm{~ms}$ were discarded ( $2 \%$ of all RT1s and $2 \%$ of all RT 2 s). Trials with an incorrect response to Task 1 (3\% of all trials) or Task 2 ( $7 \%$ of all trials) were excluded from the RT analyses. The resulting mean RTs and PEs for Task 1 and Task 2 are shown in Table 1.

RT analysis As is shown in Fig. 4 (lower panel), we obtained a PRP effect of $216 \mathrm{~ms}\left[F(3,78)=222.75, p<.01, \eta_{\mathrm{p}}{ }^{2}=.90\right]$. This cognitive slack should be long enough to absorb the Task-2 difficulty effect ( $39 \mathrm{~ms}$ at the longest SOA) $[F(1,26)=18.97$, $\left.p<.01, \eta_{\mathrm{p}}^{2}=.42\right]$.

Most importantly, the Task-2 difficulty effect decreased sharply at the shortest SOA (the difficulty effect was 6, 29, 45, and $39 \mathrm{~ms}$ at the 100, 300, 500, and 900-ms SOAs, respectively) $\left[F(3,78)=5.09, p<.01, \eta_{\mathrm{p}}{ }^{2}=.16\right]$. The difficulty effect of $6 \mathrm{~ms}$ ( $95 \%$ CI: $[-11,23])$ at the shortest SOA was significantly smaller than the 39-ms difficulty effect at the longest SOA $[F(1$, $\left.26)=6.82, p<.05, \eta_{\mathrm{p}}{ }^{2}=.21\right]$, and it was not significantly greater than $0 \mathrm{~ms}\left[F(1,26)=0.55, p=.47, \eta_{\mathrm{p}}{ }^{2}=.02\right]$. These results closely match the pattern of underadditivity of Task-2 difficulty effects across SOAs observed in Experiment 1.

$P E$ analysis There was a significant main effect of SOA on the mean PE1 $\left[F(3,78)=4.91, p<.01, \eta_{\mathrm{p}}{ }^{2}=.16\right]$ showing a PE1 
increase of .02 at the shortest SOA compared to the longest SOA. No other main effects or interactions on PE1 were statistically significant. The mean PE2 was not affected by $\mathrm{SOA}[F(3$, $\left.78)=0.68, p=.57, \eta_{\mathrm{p}}{ }^{2}=.03\right]$. However, it was affected by the Task-2 difficulty manipulation $\left[F(1,26)=39.90, p<.01, \eta_{\mathrm{p}}{ }^{2}=\right.$ $.61]$; PE2 in the easy condition was .05, and it was increased to .08 in the difficult condition. The difficult effects on PE2 were $.02, .04, .05$, and .03 at the 100-, 300-, 500-, and 900-ms SOAs, respectively; the overall interaction between difficulty and SOA was not significant $\left[F(3,78)=1.16, p=.33, \eta_{\mathrm{p}}{ }^{2}=.04\right]$.

To summarize, the results replicated the underadditivity observed in Experiment 1, with substantially smaller difficulty effects at the shortest SOA $(6 \mathrm{~ms})$ than the longest SOA (39 $\mathrm{ms})$. This finding provides further evidence that identification of familiar faces can operate automatically, while central attentional resources are devoted to another task.

\section{Experiment 3}

In Experiments 1 and 2, we used pictures of celebrities with whom participants had long-term familiarity. Can automatic identification occur with unfamiliar faces as well? Some previous studies suggest that familiar faces receive some processing advantages relative to unfamiliar faces. For example, a study using single-cell recording in macaque monkeys showed that human faces familiar to the monkeys invoked consistent neural representations, regardless of the directions of the faces, whereas unfamiliar human faces did not (Eifuku, De Souza, Nakata, Ono, \& Tamura, 2011). This finding suggests that identification is more efficient for familiar faces than unfamiliar faces. Human behavioral data also suggest that familiar faces are processed faster than unfamiliar faces for several different judgments, such as orientation, gender, and identity (Balas, Cox, \& Conwell, 2007). Also, Jackson and Raymond (2006) found that familiar T2 faces were immune to the $\mathrm{AB}$ effect (suggesting automaticity), whereas unfamiliar faces were not (but see also Awh et al., 2004).

If the familiarity of faces is essential for automatic identification, then when faces are unfamiliar to participants, we should not observe underadditivity. In this experiment, we tested this familiarity hypothesis using faces of entertainers who were not (yet) very well known to our participants. Participants first performed practice blocks of facial identification only until they achieved the accuracy criterion of $85 \%$ or they completed four practice blocks. Then they performed dual-task trials, just as in the first two experiments, to test whether unfamiliar faces can also be identified automatically.

Method

Participants A group of 65 undergraduate students from the University of New Mexico participated in exchange for course credit. All self-reported normal or corrected-tonormal visual acuity.

Apparatus and stimuli Experiment 3 was similar to Experiments 1 and 2, except that facial pictures of three much less famous entertainers were used: Jeremy Northam (actor), Keith Barry (magician), and Simon Baker (actor). An advantage of using lesser-known entertainers (rather than complete strangers) is that it serves to better match the picture characteristics (e.g., attractiveness) of those used in Experiments 1 and 2. Using the same approach described for the first two experiments, we collected four pictures of each entertainer for the easy condition and created four morphed images of each for the difficult condition.

Procedure and design In the beginning of the experiment, we checked whether participants were familiar with any of the three entertainers. We presented the four original pictures for each individual on a screen and asked the following questions: (1)“What is this person's name?”, (2)“Where have you seen him?", and (3) "How familiar does he seem?" Based on the responses, we assessed each participant's familiarity with the entertainers, using a scale from 1 to 4 . A score of 4 meant that the participant knew the name of the entertainer. A score of 3 meant that the participant did not know the name, but knew where he/she had seen him. A score of 2 meant that the participant did not know the entertainer's name or where he/she had seen him, but the entertainer seemed subjectively familiar. A score of 1 meant that the participant did not know the name or where he/she had seen him, and the entertainer did not seem very familiar. These familiarity scores were used to screen out participants who were fairly familiar with the entertainers prior to participating in our experiment, operationally defined as producing a familiarity score of 3 or 4 .

After this familiarity check, participants were allowed unlimited time to study all 12 original pictures, shown next to the corresponding first name. Then, participants completed a practice block of 24 tone-discrimination-only trials, where they responded to the tones but ignored the facial pictures. Then, we showed all 12 pictures again for as long as participants wanted to study them. Then, we gave participants faceidentification-only practice blocks ( 24 trials each with original faces only) until they reached $85 \%$ accuracy in a block, or until they had performed the maximum of four practice blocks. After each face-only practice block, participants had another opportunity to study the 12 original faces.

Results and discussion

Familiarity analysis No participant was able to name any of the three entertainers. However, we excluded data from five participants with familiarity scores of 3 for more than one of 
the entertainers, suggesting relatively high familiarity. For the remaining 60 participants, mean familiarity scores were 1.08 , 1.05, and 2.00 for Jeremy Northam, Keith Barry, and Simon Baker, respectively. Note that the minimum possible familiarity score was 1 (not 0 ), so the reported familiarity scores suggest that our participants were generally unfamiliar with the stimulus faces.

$R T$ analysis Data quality control was similar to that for the previous experiments. Trials with RT1 or RT2 below $200 \mathrm{~ms}$ or above $1,500 \mathrm{~ms}$ were discarded ( $2 \%$ of all RT1s and $2 \%$ of all RT2s). Trials with an incorrect response to Task 1 ( $2 \%$ of all trials) or Task 2 (7\% of all trials) were excluded from the RT analysis. The resulting mean RTs and PEs for Task 1 and Task 2 are shown in Table 1.

As is shown in Fig. 5 (upper panel), the mean RT2 increased by $256 \mathrm{~ms}$ as SOA decreased, showing a significant
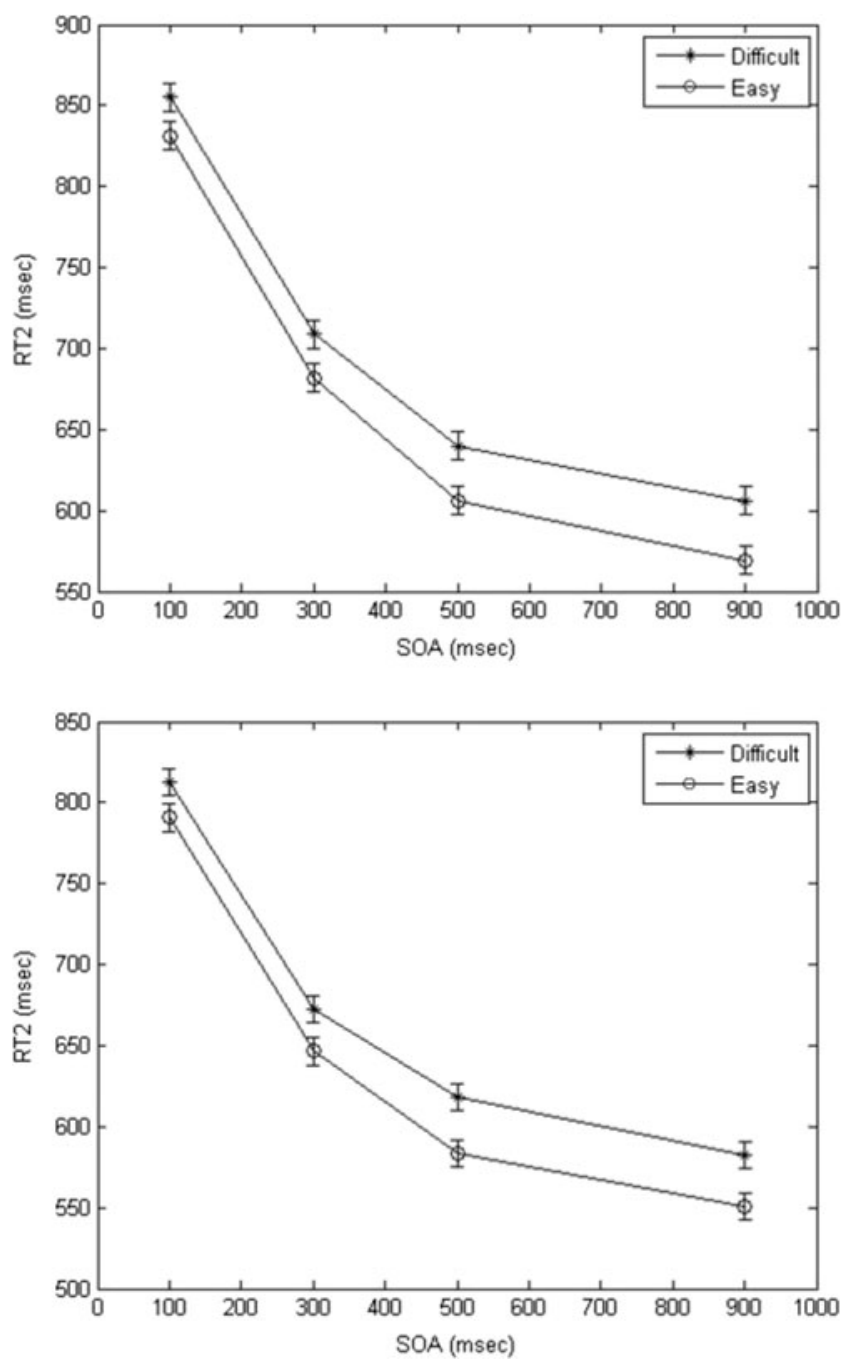

Fig. 5 Mean response times (RTs, in milliseconds) for Task 2 in Experiment 3 (upper panel) and Experiment 4 (lower panel) as a function of stimulus onset asynchrony (SOA, in milliseconds) and Task-2 difficulty
PRP effect $\left[F(3,177)=426.74, p<.01, \eta_{\mathrm{p}}{ }^{2}=.88\right]$. Critically, the Task-2 difficulty effect on RT2 did not vary significantly across SOAs $[F(3,177)=1.40, p=.25$, $\left.\eta_{\mathrm{p}}{ }^{2}=.02\right]$; the difficulty effects were $23,27,34$, and $37 \mathrm{~ms}$ at the 100-, 300-, 500-, and 900-ms SOAs, respectively. The difficulty effect of $23 \mathrm{~ms}(95 \% \mathrm{CI}:[8,38])$ at the shortest SOA was significantly greater than $0 \mathrm{~ms}[F(1,59)=9.61$, $\left.p<.01, \eta_{\mathrm{p}}{ }^{2}=.14\right]$, and it was not statistically different from the $37-\mathrm{ms}$ difficulty effect at the longest SOA, although it approached significance $\left[F(1,59)=3.02, p=.09, \eta_{\mathrm{p}}^{2}=.05\right]$. The substantial difficulty effect at the shortest SOA suggests that, when participants do not have long-term familiarity with target faces, they have difficulty identifying the faces automatically.

$P E$ analysis We found a significant main effect of SOA on the mean PE1 $\left[F(3,177)=21.60, p<.01, \eta_{\mathrm{p}}{ }^{2}=.27\right]$, with a PE1 increase of .03 at the shortest SOA as compared to the longest SOA. No other main effects or interactions regarding PE1 were statistically significant. There was a significant Task-2 difficulty effect on PE2 $\left[F(1,59)=54.83, p<.01, \eta_{\mathrm{p}}{ }^{2}=.48\right]$; the difficult effect on PE2 in the easy condition was .06, and it increased to .09 in the difficult condition. No other tests regarding PE2 showed significant results.

To summarize, the present experiment was designed to evaluate the hypothesis that long-term familiarity with faces is critical for automatic facial identification. Consistent with this hypothesis, we did not see a strong and significant underadditive pattern between difficulty and SOA.

\section{Experiment 4}

In Experiment 3, Simon Baker was unfamiliar to most participants, but his familiarity rating of 2.00 was somewhat higher than the other two entertainers. To reduce the overall familiarity level even further, we replaced his facial pictures with those of Casey Affleck and replicated the experiment.

Method

Participants A group of 46 undergraduate students from the University of New Mexico participated in exchange for course credit. All self-reported normal or corrected-to-normal visual acuity.

Apparatus, stimuli, procedure, and design Experiment 4 was similar to Experiment 3, except that Simon Baker's face pictures were replaced by Casey Affleck's pictures. Likewise, eight new morphed faces were created for the difficult condition by morphing Casey Affleck's pictures with those of Jeremy Northam and Keith Barry. Hence, among the 24 pictures used in this experiment, half were new (four Casey Affleck pictures and eight newly morphed 
images). The same familiarity check and face learning procedures described in Experiment 3 were also used in Experiment 4.

\section{Results and discussion}

Familiarity analysis Four of the participants were able to name Casey Affleck from his pictures. We excluded their data from further data analysis. We also excluded the data from five participants with familiarity scores of 3 for more than one of the entertainers. For the remaining 37 participants, mean familiarity scores were 1.18, 1.05, and 1.58 for Jeremy Northam, Keith Barry, and Casey Affleck, respectively.

$R T$ analysis Data quality control was similar to that for the previous experiments. Trials with RT1 or RT2 below $200 \mathrm{~ms}$ or above $1,500 \mathrm{~ms}$ were discarded (5\% of all RT1s and $1 \%$ of all RT2s). Trials with an incorrect response to Task 1 (2\% of all trials) or to Task 2 (6\% of all trials) were excluded from RT analysis. We discarded the data from one participant with mean Task-2 accuracy in the easy condition below our criterion of $80 \%$. In all, 36 out of the 46 participants remained for further data analysis. The resulting mean RTs and PEs for Task 1 and Task 2 are shown in Table 1.

As is shown in Fig. 5 (lower panel), the mean RT2 showed a substantial PRP effect of $235 \mathrm{~ms}[F(3,105)=299.10, p<.01$, $\left.\eta_{\mathrm{p}}{ }^{2}=.90\right]$. The Task-2 difficulty effect on RT2 did not vary significantly across SOAs $[F(3,105)=0.82, p=.49$, $\left.\eta_{\mathrm{p}}{ }^{2}=.02\right]$; the difficulty effects were $22,26,34$, and $32 \mathrm{~ms}$ at the 100-, 300-, 500-, and 900-ms SOAs, respectively. The difficulty effect of $22 \mathrm{~ms}(95 \% \mathrm{CI}$ : $[5,39])$ at the shortest SOA was significantly greater than $0 \mathrm{~ms}[F(1,35)=7.26, p<.05$, $\left.\eta_{\mathrm{p}}{ }^{2}=.17\right]$, and it was not statistically different from the 32-ms difficulty effect at the longest $\mathrm{SOA}[F(1,35)=1.54, p=.22$, $\left.\eta_{\mathrm{p}}{ }^{2}=.04\right]$. The nearly additive pattern of Task- 2 difficulty effect across SOAs is consistent with that of Experiment 3 and further supports the conclusion that recently learned faces cannot be identified automatically.

$P E$ analysis A significant main effect of SOA on mean PE1 emerged $\left[F(3,105)=6.37, p<.01, \eta_{\mathrm{p}}{ }^{2}=.15\right]$, with a PE1 increase of .02 at the shortest relative to the longest SOA. No other main effects or interactions were statistically significant for PE1. We found a significant Task-2 difficulty effect on PE2 $\left[F(1,35)=27.98, p<.01, \eta_{\mathrm{p}}{ }^{2}=.44\right]$; the PE2 in the easy condition was .05 , and it increased to .07 in the difficult condition. No other effects were statistically significant for PE2.

To summarize, this experiment replicated the main finding of Experiment 3 that unfamiliar faces were not processed completely automatically. The difficulty effect remained substantial and statistically significant at the shortest SOA (unlike Experiments 1 and 2, where it disappeared).

\section{General discussion}

The present study used the PRP paradigm to investigate whether face identification is automatic, in the sense that it does not require central attentional resources. In the present experiments, participants attempted to identify faces (as Task 2) while performing a concurrent tone task (Task 1). In Experiment 1, we used a set of three highly familiar celebrity faces. Task-2 difficulty effects were completely underadditive with SOA, which, according to locus-ofslack logic, indicates that facial identification is automatic. In Experiment 2, we used a new set of celebrity faces to test whether the observed underadditivity in Experiment 1 was a stimuli-specific phenomenon. Again, we found the same strong underadditive pattern. The data are consistent with the hypothesis that identification of familiar faces can proceed at full speed, even while central attentional resources are devoted to another demanding task.

The automatic face identification observed in Experiments 1 and 2 is somewhat surprising. Previous PRP studies using facial stimuli suggest that other facial information processes with a seemingly similar level of complexity such as facial emotion perception (Tomasik et al., 2009; but see also Shaw et al., 2011) or facial attractiveness judgments (Jung et al., 2012) are not automatic. Those experiments produced additive interactions between SOA and difficulty, not underadditive. Given these results, one could speculate that facial identification has even higher adaptive importance than the other facial information processes. Or, facial identification might be more time-critical, placing greater pressure to develop complete automaticity. One could also speculate that facial identification might have a greater level of practices than the other facial processes. Another possibility, however, is that the present Experiments 1 and 2 used faces familiar to participants, whereas previous studies of facial emotion and attractiveness have used unfamiliar faces. Further research will be needed to determine whether emotion perception and attractiveness judgments would be automatic for familiar faces.

The role of familiarity in automatic facial identification

Whereas Experiments 1 and 2 (with highly familiar faces) showed complete underadditivity, suggesting full automaticity, Experiments 3 and 4 (with unfamiliar faces) did not. To verify that the results differ significantly between these sets of experiments, we conducted an additional betweenexperiment analysis. For this test, we compared the pooled the data from Experiments 1 and 2 to the pooled data from Experiments 3 and 4 . The analysis was a repeated measures ANOVA with Difficulty and SOA (shortest and longest only) as within-subjects factors and Experiment Type (Exps. 1 and 2 vs. Exps. 3 and 4) as a between-subjects factor. Critically, we identified a statistically significant 
three-way interaction between difficulty, SOA, and experiment type $\left[F(1,165)=5.85, p<.05, \eta_{\mathrm{p}}{ }^{2}=.03\right]$. This interaction indicates that the amount of underadditivity observed in Experiments 1 and 2 was significantly larger than the slight underadditivity in Experiments 3 and 4. Therefore, this result supports the hypothesis that long-term familiarity facilitates automatic facial identification.

Inspection of Fig. 5 suggests a slight underadditive trend from Experiment 3 and 4, with unfamiliar faces. To test whether this trend indicating partial automaticity is real, we conducted a follow-up analysis pooling data from both experiments. The combined difficulty effects were $23,26,34$, and $35 \mathrm{~ms}$ at the 100-, 300-, 500-, and 900-ms SOAs, respectively. This slight underadditive trend was not significant in the ANOVA that included all SOAs $[F(3,285)=2.16, p=.10$, $\left.\eta_{\mathrm{p}}^{2}=.022\right]$, although it reached significance in a more specific analysis comparing just the two extreme SOAs (i.e., 100 and $900 \mathrm{~ms})\left[F(1,95)=4.56, p=.04, \eta_{\mathrm{p}}{ }^{2}=.05\right]$. Although the $34 \%$ reduction in difficulty effects suggests partial automaticity, it is not full automaticity because the difficulty effect of $23 \mathrm{~ms}$ ( $95 \%$ CI: $[12,34])$ at the shortest SOA was significantly greater than $0 \mathrm{~ms}\left[F(1,95)=16.73, p<.05, \eta_{\mathrm{p}}{ }^{2}=.15\right]{ }^{4}$

Given that we studied a finite number of famous and less famous faces, it is logically possible that the differential automaticity reflects potential (unintended) differences in picture characteristics, not familiarity per se. For instance, the celebrity pictures used in Experiments 1 and 2 could have more distinctive cues, such as hairstyles or facial expressions, and it is possible that participants used such cues to short-cut the identification process. We deliberately tried to avoid such confounds by choosing four different face pictures for each entertainer, and by requiring that all pictures had specific characteristics, such as minimal emotion, mostly closed mouths, and facing the camera. Furthermore, the results were replicated with more than one set of celebrity pictures. Nevertheless, to indirectly test this alternative explanation, we examined the overall speed of facial identification at the longest SOAs. We found that the mean face-identification RT actually tended to be slightly longer for the more famous celebrities than the less famous ones ( 611 vs. $577 \mathrm{~ms}$ ), $[t(165)=3.13, p=.02]$. This result suggests that participants

\footnotetext{
$\overline{4}$ The slight underadditivity in Experiments 3 and 4 could have been a result of the increased familiarity from early to late experimental blocks (Blocks 2-6 vs. Blocks 8-12). To better understand the impact of increasing familiarity from early to late experimental blocks, a follow-up analysis was conducted, pooling data from both experiments. The difficulty effects across the four SOAs were as follows: $26,19,33$, and $34 \mathrm{~ms}$, respectively, for the earlier blocks, and 15, 35, 32 , and $36 \mathrm{~ms}$ for the later blocks. The apparent trend toward more underadditivity in later blocks did not reach significance $[F(1,190)=$ $0.90, \mathrm{p}=.35, \eta \mathrm{p} 2=.01]$. The present study included only a modest range of practice levels; to address this issue more thoroughly, further research will be needed, with greater amounts of practice.
}

were not relying on specific low-level image cues with the familiar faces to identify them.

Automatic identification of familiar faces, but not unfamiliar ones, is consistent with the results of some previous studies. For example, Buttle and Raymond (2003) observed that changes between two successive faces could be more easily detected when the target face was familiar to participants. Similarly, Tong and Nakayama (1999) showed that extremely familiar faces, such as one's own face, can be detected much more quickly when embedded amongst unfamiliar faces rather than familiar ones.

Interestingly, Reddy, Reddy, and Koch (2006) showed that, regardless of the familiarity level, faces can be recognized in the near-absence of focal attention. In their study, participants performed a letter discrimination task that requires focal visual attention, and an identification task on peripherally presented faces. The two tasks were performed either alone or concurrently. Their results showed that, regardless of the familiarity level, faces can be recognized in the absence of visual attention, whereas other kinds of identification (e.g., identifying artificial geometric stimuli such as bisected red-green disks) cannot. The difference in results between their study and ours might reflect the different types of attentional resources being studied: visual attention to locations versus central attention to tasks. Combining the study with ours, we can summarize that all faces can be identified without fully available visual attention, but only familiar faces can be identified without central attentional resources. This leads us the following question that deserves further study: whether peripherally presented familiar faces can be identified automatically.

Potential sources of automatic identification of familiar faces

As we noted above, faces provide information critical to survival and reproductive fitness, which might explain the present evidence for automatic identification of familiar faces. Automatic identification of familiar faces would help people to respond appropriately and rapidly to the appearance of a familiar person (e.g., family, friend or enemy), even when they are already engaged in deep thought about something else. Consistent with this view, certain areas of the brain, especially the so-called face area, selectively respond to faces over other types of stimuli (e.g., Kanwisher, McDermott, \& Chun, 1997). However, this finding does not by itself explain why we observed greater evidence of automaticity from familiar faces than from unfamiliar ones. Several authors have proposed that the face area is actually responsible for expert object recognition (i.e., facilitating the processing of all stimuli with which participants have great expertise; Tarr \& Gauthier, 2000). Accordingly, the key to automatic identification of familiar faces might be perceptual expertise or high enough familiarity level obtained from practices, rather than some special (evolutionary) advantages for faces in general. One direction 
for future research is to determine whether identification of equally complex stimuli to faces but of little evolutionary importance (e.g., birds or cars) can become automatic for experts of those domains. If experts can identify birds or cars automatically, then practice itself (without any evolutionary motivation) would suffice to cause automaticity.

Finally, familiarity with faces is also known to help us to overcome degradations of facial images (i.e., low-resolution images) during identification. For example, Burton, Wilson, Cowan, and Bruce (1999) showed that observers' face recognition performance with low-quality surveillance video is much better when the observers were already personally familiar with the target individual then when they had interacted infrequently with that individual. Notably, police officers with experience in forensic identification performed as poorly as the observers who were unfamiliar with the target individuals. However, exactly how we establish familiarity or expertise to faces is as yet unclear. It might be the result of a large number of stored exemplars (Bahrick, Bahrick, \& Wittlinger, 1975). For instance, the more exemplars that one has stored of Brad Pitt, the more likely there will be a near-perfect match to the presented face. Another possibility is that the familiar faces have much stronger associations with the identity of the person, resulting in automatic facial identification without help from central attention resources.

\section{Concluding remarks}

We conclude that facial identification is not fully automatic for unfamiliar faces. However, as we become more familiar with faces, identification appears to become fully automatic. This automaticity is impressive, because faces are very complex objects, and many simpler perceptual operations have proven to be nonautomatic. The automaticity of familiar-face identification might stem from high practice levels, or high evolutionary importance, or the combination of the two.

\section{References}

Allison, T., Puce, A., Spencer, D. D., \& McCarthy, G. (1999). Electrophysiological studies of human face perception. I: Potentials generated in occipitotemporal cortex by face and nonface stimuli. Cerebral Cortex, 9, 415-430.

Awh, E., Serences, J., Laurey, P., Dhaliwal, H., van der Jagt, T., \& Dassonville, P. (2004). Evidence against a central bottleneck during the attentional blink: Multiple channels for configural and featural processing. Cognitive Psychology, 48, 95-126. doi:10.1016/S0010-0285(03)00116-6

Bahrick, H. P., Bahrick, P. O., \& Wittlinger, R. P. (1975). Fifty years of memory for names and faces: A cross-sectional approach. Journal of Experimental Psychology: General, 104, 54-75.

Balas, B., Cox, D., \& Conwell, E. (2007). The effect of real-world personal familiarity on the speed of face information processing. PLoS ONE, 2, e1223. doi:10.1371/journal.pone.0001223
Bauer, R. M. (1984). Autonomic recognition of names and faces in prosopagnosia: A neuropsychological application of the guilty knowledge test. Neuropsychologia, 22, 457-469.

Boutet, I., Gentes-Hawn, A., \& Chaudhuri, A. (2002). The influence of attention on holistic face encoding. Cognition, 84, 321-341.

Bruce, V. (1979). Searching for politicians: An information-processing approach to face recognition. Quarterly Journal of Experimental Psychology, 31, 373-395.

Bruce, V., \& Young, A. (1986). Understanding face recognition. British Journal of Psychology, 77, 305-327.

Burton, A. M., Wilson, S., Cowan, M., \& Bruce, V. (1999). Face recognition in poor-quality video. Psychological Science, 10, 243-248.

Buttle, H., \& Raymond, J. E. (2003). High familiarity enhances visual change detection for face stimuli. Perception \& Psychophysics, 65, 1296-1306.

Eifuku, S., De Souza, W. C., Nakata, R., Ono, T., \& Tamura, R. (2011). Neural representations of personally familiar and unfamiliar faces in the anterior inferior temporal cortex of monkeys. PLOS ONE, 6, e18913. doi:10.1371/journal.pone.0018913

Farah, M. J., Wilson, K.-D., Drain, M., \& Tanaka, J. N. (1998). What is "special" about facial perception? Psychological Review, 105, 482-498. doi:10.1037/0033-295X.105.3.482

Grammer, K., Fink, B., Møller, A. P., \& Thornhill, R. (2003). Darwinian aesthetics: Sexual selection and the biology of beauty. Biological Reviews, 78, 385-407.

Jackson, M. C., \& Raymond, J. E. (2006). The role of attention and familiarity in face identification. Perception \& Psychophysics, 68 , 543-557. doi:10.3758/BF03208757

Johnston, J. C., \& McCann, R. S. (2006). On the locus of dual-task interference: Is there a bottleneck at the stimulus classification stage? Quarterly Journal of Experimental Psychology, 59, 694-719.

Jung, K., Ruthruff, E., Tybur, J., Gaspelin, N., \& Miller, G. (2012). Perception of facial attractiveness requires some attentional resources: Implications for the "automaticity" of psychological adaptations. Evolution and Human Behavior, 33, 241-250.

Kanwisher, N., McDermott, J., \& Chun, M. M. (1997). The fusiform face area: A module in human extrastriate cortex specialized for face perception. Journal of Neuroscience, 17, 4302-4311.

Khurana, B., Smith, W. C., \& Baker, M. T. (2000). Not to be and then to be: Visual representation of ignored unfamiliar faces. Journal of Experimental Psychology: Human Perception and Performance, 26, 246-263. doi:10.1037/0096-1523.26.1.246

Langton, S. R. H., Watt, R. J., \& Bruce, V. (2000). Do the eyes have it? Cues to the direction of social attention. Trends in Cognitive Sciences, 4, 50-59.

Lavie, N., Ro, T., \& Russell, C. (2003). The role of perceptual load in processing distractor faces. Psychological Science, 14, 510-515.

Levy, J., Pashler, H., \& Boer, E. (2006). Central interference in driving: Is there any stopping the psychological refractory period? Psychological Science, 17, 228-235.

Lien, M.-C., Allen, P. A., Ruthruff, E., Grabbe, J., McCann, R. S., \& Remington, R. W. (2006a). Visual word recognition without central attention: Evidence for greater automaticity with advancing age. Psychology and Aging, 21, 431-447.

Lien, M.-C., Ruthruff, E., \& Johnston, J. C. (2006b). Attentional limitations in doing two things at once: The search for exceptions. Current Directions in Psychological Science, 15, 89-93.

Liu, J., Harris, A., \& Kanwisher, N. (2002). Stages of processing in face perception: An MEG study. Nature Neuroscience, 5, 910-916.

Maquestiaux, F., Laguë-Beauvais, M., Ruthruff, E., \& Bherer, L. (2008). Bypassing the central bottleneck after single-task practice in the psychological refractory period paradigm: Evidence for task automatization and greedy resource recruitment. Memory \& Cognition, 36, 1262-1282. doi:10.3758/MC.36.7.1262 
Norman, D. A., \& Bobrow, D. G. (1975). On data-limited and resource-limited processes. Cognitive Psychology, 7, 44-64. doi:10.1016/0010-0285(75)90004-3

O’Malley, S., Reynolds, M. G., Stolz, J. A., \& Besner, D. (2008). Reading aloud: Spelling-sound translation uses central attention. Journal of Experimental Psychology: Learning, Memory, and Cognition, 34, 422-429.

Palermo, R., \& Rhodes, G. (2002). The influence of divided attention on holistic face perception. Cognition, 82, 225-257.

Palermo, R., \& Rhodes, G. (2007). Are you always on my mind? A review of how face perception and attention interact. Neuropsychologia, 45, 75-92.

Pashler, H. (1984). Processing stages in overlapping tasks: Evidence for a central bottleneck. Journal of Experimental Psychology: Human Perception and Performance, 10, 358-377.

Pashler, H., \& Johnston, J. C. (1989). Chronometric evidence for central postponement in temporally overlapping tasks. Quarterly Journal of Experimental Psychology, 41, 19-45.

Reddy, L., Reddy, L., \& Koch, C. (2006). Face identification in the near-absence of focal attention. Vision Research, 46, 2336-2343.

Ruthruff, E., Allen, P. A., Lien, M.-C., \& Grabbe, J. (2008). Visual word recognition without central attention: Evidence for greater automaticity with greater reading ability. Psychonomic Bulletin \& Review, 15, 337-343. doi:10.3758/PBR.15.2.337

Ruthruff, E., Johnston, J. C., \& Remington, R. W. (2009). How strategic is the central bottleneck: Can it be overcome by trying harder? Journal of Experimental Psychology: Human Perception and Performance, 35, 1368-1384.

Ruthruff, E., Miller, J. O., \& Lachmann, T. (1995). Does mental rotation require central mechanisms? Journal of Experimental
Psychology: Human Perception and Performance, 21, 552570.

Schneider, W., \& Fisk, A. D. (1982). Degree of consistent training: Improvements in search performance and automatic process development. Perception \& Psychophysics, 31, 160-168.

Schneider, W., \& Shiffrin, R. M. (1977). Controlled and automatic human information processing: I. Detection, search, and attention. Psychological Review, 84, 1-66. doi:10.1037/0033-295X.84.1.1

Shaw, K., Lien, M.-C., Ruthruff, E., \& Allen, P. A. (2011). Electrophysiological evidence of emotion perception without central attention. Journal of Cognitive Psychology, 23, 695-708.

Shiffrin, R. M., \& Schneider, W. (1977). Controlled and automatic human information processing: II. Perceptual learning, automatic attending and a general theory. Psychological Review, 84, 127190. doi:10.1037/0033-295X.84.2.127

Stone, A. M., \& Valentine, T. (2004). Better the devil you know? Nonconscious processing of identity and affect of famous faces. Psychonomic Bulletin \& Review, 11, 469-474.

Tarr, M. J., \& Gauthier, I. (2000). FFA: A flexible fusiform area for subordinate-level visual processing automatized by expertise. Nature Neuroscience, 3, 764-769.

Tomasik, D., Ruthruff, E., Allen, P. A., \& Lien, M.-C. (2009). Nonautomatic emotion perception in a dual-task situation. Psychonomic Bulletin \& Review, 16, 282-288. doi:10.3758/PBR.16.2.282

Tong, F., \& Nakayama, K. (1999). Robust representation of faces: Evidence from visual search. Journal of Experimental Psychology: Human Perception and Performance, 25, 1016-1035.

Tranel, D., \& Damasio, A. R. (1985). Knowledge without awareness: An autonomic index of facial recognition by prosopagnosics. Science, 228, 1453-1454. 